\title{
IoT-smart contracts in data trusted exchange supplied chain based on block chain
}

\author{
S. Ganesh Kumar, B. Sriman, A. Murugan, B. Muruganantham \\ Department of Computer Science and Engineering, Faculty of Engineering and Technology, \\ SRM Institute of Science and Technology, Chennai, India
}

\begin{abstract}
Article Info
Article history:

Received Feb17, 2019

Revised Aug 23, 2019

Accepted Aug 30, 2019

Keywords:

Blockchain

Datatrusted exchange

IoT (internet of things)

Smart contracts

ABSTRACT

Internet of Things (IoT) assumes a critical part in the advancement of different fields. The IoT data trusted exchange in recent year extend of uses influence an awesome request and increasing scale. In such a platform, exchange the data sets that they require and specialist organization can search. However, the enough trust as the third-party mediators for data exchange in centralized infrastructure cannot provide. This paper proposes a blockchain for IoT data trusted exchange based on decentralized solution. In particular, the fundamental standards of blockchain in verify manner, individuals can communicate with each other without a confided in mediator intermediary. Blockchain enable us to have a distributed, digital ledger. IoT (Internet of Things) sensor devices (zigbee) utilizing blockchain technology to assert public availability of temperature records, tracking location shipment, humidity, preventing damage, data immutability. The sensor devices looking the temperature, location, damage of each parcel during the shipment to completely guarantee directions. In blockchain all data is got moved from one position to another, where a smart contract assesses against the product attributes. Ethereum blockchain and smart contracts atlast it gets through knowledge a design to be copied and presents its decentralized distributed digital ledger, auditable, transparent, features visually.
\end{abstract}

Copyright $\odot 2020$ Institute of Advanced Engineering and Science. All rights reserved.

\section{Corresponding Author:}

S. Ganesh Kumar,

Department of Computer Science and Engineering,

Faculty of Engineering and Technology,

SRM Institute of Science and Technology,

Chennai, India.

Email: 13ganesh@mail.com

\section{INTRODUCTION}

Internet of Things (IoT) and Blockchain are viewed as rising ideas and technologies [1]. In the meantime they change ideas and make new conceivable outcomes, each in their particular situations, and there is a chance to make applications that can share the inborn attributes of both, investigating how the IoT can profit by the decentralized idea of the Blockchain. The forward development of exchange and networking technologies (e.g., Wi-Fi, Zigbee, Bluetooth), a developing more complete number of things (e.g., sensors, actuators, smart devices) are being associated Internet these days, are being connected to the Internet these day (IoT).

Blockchain [2-5] basically a distributed, digital ledger [6] has numerous applications and can be used for any data exchange, agreements/contracts, tracking and of course, payment [6,7]. Since every bit of transaction is recorded on a block and across number times another copies of the ledger that are made distribution over many nodes (computers), it is highly transparent. It's also profoundly safe since each block makes connection to the one going in front of it and after it. There is not one focal being of the opinion that over the blockchain [2], and it's to a great degree working well and turning readily to another work. 
In the end, blockchain can make the power and transparency of supply chains and decision act on everything from warehousing to delivery taken to payment. Chain of need is basic for some things, and blockchain has the chain of need made in. Gained recently attention with [8] Smart contracts, particularly with in connection with to the blockchain technology.

Selected before the rules, blockchain technology that can verify [9] its correctness and support in smart contract (agreement) hence, its a self-implementation and self- executing. However, at all base platform without a right base platform a smart contract is not "smart" to run, execute and check these contracts it need such a base platform. A smart contracts that can work in a decentralized manner and completely self-ruling, such a base platform is blockchain. Financial services [10] (e.g., Bitcoin) or general services e.g., (Ethereum) [11, 12] can be used smart contract. A blockchain [4] executes, checks, and gathers and stores smart contracts in blocks. Every block has a statement, direction to atleast one person who had the position before, for this reason the limited stretch of time blockchain [13]. Blockchains are decentralized, distribute ledger, [7] based on cryptograph. A main interest in the financial industry for using blockchains is to put machines to use and digitalizing forms particularly when great number of a number of persons working together are covered. These agreement can be assessed naturally smart contract with blockchain utilizing the primary advantage.

Current solutions produce that needs to be checked manually using smart contracts, the temperature indicators, tracking shipping, preventing shipping damage can be assesses automatically and notify sender and one who gets package. In addition, it is tamper-proof for the stored data and are used for looking over of account by expert by outside groups of persons. By using the Ethereum [11, 12], it is fully decentralized as tamper-proof, framework utilized by the requiring little to no hard work and on a for every contract and per byte premise.

The remaining sections will explain the upcoming topics. The second part, Internet of Things (IoT) zigbee based technology wireless sensor network (WSN) discovered shipping damage indicators, temperature indicators and tracking shipping. Section III Blockchain enables the work of art of smart contracts, with terms and conditions both sides can give details and that say without doubt have trust in the enforceability of the contract and the mind and physical qualities of the counterparty. Section IV outlines the special technical details, which is had followed by a first stage put value evaluation, while Section V provides conclusions.

\section{ZIGBEE BASED TECHNOLOGY (WSN)}

Many applications that used in the great numbers today are GPS. Tracking is one of the applications in shipping or Any Portable Device and monitored regularly. The routs travelled and the exact locations of the information given by this tracking system, which are embedded in this hardware. Moreover, by that given information the user can locate the different places widely. In any of the weather conditions, the systems are enabled to track the target routes. For this, the Zigbee technology and the GPS are used. Similar to this, the landwide shipment tracking used similar to the GPS is represented. This type of the systems enable at the same time for the required Equipment's to be tracked, which are accurate, long lasting, light in weight and are cheaper than any of the automatic positioning tags. In any devices, the sensors are built into the compactness of the prototypes, which are open structure designed for tracking the shipments. By using the Google Maps, the GPS and the API working: the information are sent over the network to devices like mobile phones which are embedded with the simple Zigbee technology for tracking the device shipments. Whenever, it is limiting to the particular person with an adjusting alert message to the receiver for tracking. The battery powers are saved and the tracking costs and the feasibility results and then doing the power efficiency of the battery and the transmission of the data. Now a day's technology is growing higher and higher good level, because of this, the common people are ready to take up these technology facilities in their daily life. In their day to day living groups of person are demanding to protect their instruments, devices etc. by using available resources. Hence this project is made on the platform of this demand. Required components are:

1. Arduino.

2. GSM GPS Module.

3. $16 \times 2$ LCD.

4. Power Supply.

5. Connecting Wires.

6. Zigbee 


\subsection{Arduino UNO ATmega328}

Microcontroller board based on the AT mega328 (data sheet), hardware, software with the open source computers by the Arduino Uno. It consists of the 14 numbers with the digital input/output pins (6 used as PWM outputs), 6 inputs as the analogs, and a $16 \mathrm{MHz}$ ceramic resonator, with the power jack, an ICSP header, USB connection and the button to start. The support for the micro controller provided, to make connection to the knowledge for processing machine to an USB subscription, or turn it on with the AC to DC for making the adjustment connector for producing electric current to initiate. It uses the FTDI USB to serial driver chip, so only it is different from all the process boards. Inspite of this, it uses the Atmega 16U2 (Atmega8U2 up to R2) of the knowledge processing machine orders which are listed as USB to the serial converter. Figure 1 show the arduino UNO R3.

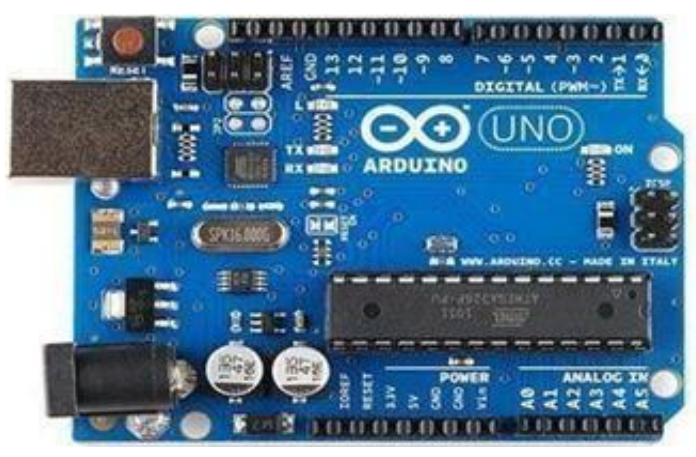

Figure 1. Arduino UNO R3

The Uno board that has a resistor version of Revision 2 that pulls the 8U2 HWB that get onto the land, which makes it easy to put them into the DFU of most frequent number. The board version of Revision 3 has the new upcoming features. The SCL pins are near to the RESET pin, 1.0 pinout: added the SDA [14] and the new other two pins are safely placed near the RESET pin. The safety shields are allowed to adjust to transmute the voltage that are provided from the board by the IOREF. The safety shields that are able to exist together are AVR used by the boards that operates with 5V and with Arduino [14] duo which are operated with 3.3V. The remaining pins are kept unconnected for the later purpose.

a. The circuit is stronger in RESET.

b. ATmega [14] 16U2 that gives another in place of $8 \mathrm{U} 2$.

"Uno" is a way one in Italian. The direction accounts of Arduino, moving forward in the Uno and version 1.0. The Uno is the latest in a number, order, group, line of USB Arduino boards, and the statement, direction scaled copy model for the Arduino platform.

\subsection{GPS-GSM module (SIM 808)}

GPS-GSM[15] part of a greater module unit (SIM808) part of a greater module unit is a wellconstructed complete Quad- Band GSM/GPRS part of a greater module unit which grain processing machines combines GPS technology for one dependent on keeping satellite navigation. The package which mixed together GPRS and GPS in a SMT will importantly for both cost and time application enabled GPRS growth develop to customers. GPS purpose use, it let not fixed in level properties to be with ways, roads, lines without breaks tracking at any place and any time with amount signal coverage in feature an industry.

\subsection{LCD 16x2}

LCD [16] (Liquid Crystal Display) is discover a wide range applications and screen is an electronic exhibit module. A 16x2 LCD is used in different circuits and device and basic part of greater module unit is exhibit. These parts of a greater module unit are supported over seven part and othermore than one or two part LEDs. The reasons being: LCDs are money related; easily able to be made into list of machine orders; have no limiting condition of displaying special \& even tax on goods coming into country characters (unlike in seven part), animations and so on. Figure 2 show LCD 16x2. The receiver part of a greater module unit is activated when its within the range of transmitter part of a greater module unit. Which is decoded and send message to the LCD [16] screen (Pin D4 to D7) to display the true statement message (Part of a greater Module unit is Found). If the receiver is in the range of transmitter part of a greater module unit then Zigbee module activated and sends the true statement signal to Arduino (Pin 2 DATA to D12). 


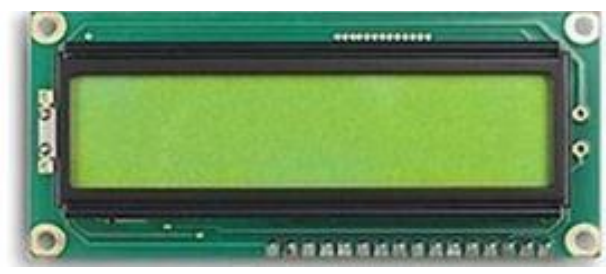

Figure 2. LCD $16 * 2$

\subsection{Power supply}

Power supply device for the make into different sort of ready (to be used) power of one group of qualities to have meeting with given details of requirements of a certain sort power supplies of application getting changed includes raw controlling the input power and/or operation of current for the electronic equipment and/or made voltage fixed.

\subsection{Zigbee}

Zigbee [15] is a wireless communication standard for low cost, low rate, low power, which can to be used far away, widely different control applications, likes smart home automation, smart cities, smart packing, smart health care system as shown in Figure 3. Zigbee quality example has been designed to offer least possible, recorded price and power to make connections for devices which have need of electric current from several years, and several months life for time ranging. Zigbee has based on the RF general condition to the looked on to come to cover 10-70 meters and given application output is required. The three main components are in Zigbee network as shown in Figure 4 zigbee based networks like routers (ZR) and person giving directions (ZC), End-devices (ZED) [15].

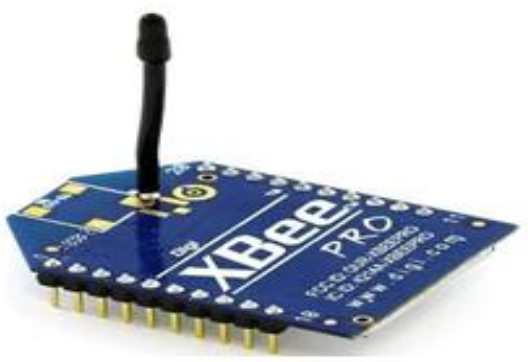

Figure 3. Zigbee

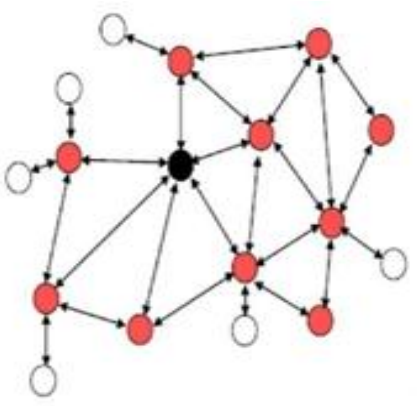

Figure 4. Zigbee based networks

a. $\mathrm{GHz}$ Radio frequency band.

b. $250 \mathrm{kbit} / \mathrm{s}$ Data rate.

c. 16 (802.15.4 Channels 11 to 26) Number of channels.

d. 2 analogue $\mathrm{I} / \mathrm{O}$ ports and 12 general purpose $\mathrm{I} / \mathrm{O}$ port inputs.

e. 100-300 meters Typical distances.

A network component is done or not in the router. In participates and coordinator it may associate with in the message of multi- hop routing. Makes connection to one person giving directions or router and low power operation which made for end-device in finally. Each Zigbee network need only one person giving directions and it starts the network structuring. Zigbee is to guide and get fixed by the signing the Detected location when the receiver part of a greater module unit is in the range of Zigbee transmitter. The GSM send the put into signs co-ordinates to Cell phone and comes back a sign put out to [16] Arduino (Pin D0) for the make up of operation completed. At the same time the Arduino activate the Zigbee transmitter part of a greater module unit (Pin D12) [17] till the operation completed and provides the $5 \mathrm{~V}$ supply. The receiver part of a greater module unit is activated when it's within the range of transmitter part of a greater module unit. Which is decoded and send message to the LCD screen (Pin D4 to D7) [17] to display the true statement message (Module is Found).

If receiver is in the range of transmitter part of a greater module unit then Zigbee part of a greater module unit activated and sends the true statement sign put out to Arduino (Pin 2 DATA to D12).

IoT-smart contracts in data trusted exchange supplied chain based on block chain (S. Ganesh Kumar) 
We can unbroken brands over wheels the taker (property of another) readily or any device by coming here after sensed co-ordinates (Latitude and longitude) and make clear the location by detecting the separate Zigbee sign put out received from the taken (property of another) device. Zigbee Wireless Network: This is the part which physically doesn't currently in existence. It is chiefly of the wireless communication between the Zigbee part of a greater modules attached to the Transmitter and Receiver Arduino board and microcontroller board.

\section{BLOCKCHAIN IN SUPPLY CHAIN MANAGEMENT AND LOGISTICS}

The essential fields in Block chain appropriation nowdays is the Logistics and the supplied chain industry [5, 7, 18]. Different enormous are looking into the implementation of blockchain [2, 3] for the easy communication process of delivers and makes the supply chain [10] traceable and efficient. This changing technology is very much helpful for tamper-proof, and tracking the product of anything begins from tomatoes to diamonds [4]. From order tracking to dispute resolution, blockchain has the response to every problem that has been plaguing the logistics industry for long time. Figure 5 shown Supply chain management in blockchain.

The information flow in current goods is highly complicated, includes many parties, and includes heavy documentation [19] (payments, receipts, settlements, etc.). Monitoring every single exchanges and documents is a cumbersome job and sometimes important documents gets of transparency in the present supply chain system. Also it's extremely difficult to investigate if there is happening of any illegal or dishonest practices in the system lost or manufactured, which creates confusion in the system, leading to huge loss.

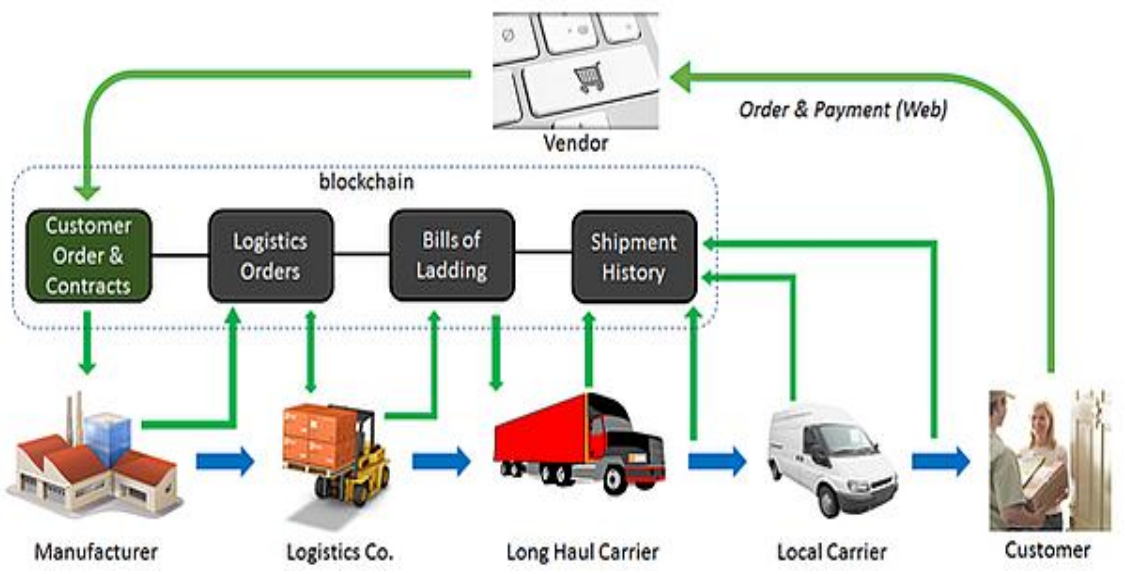

Figure 5. Supply chain management in blockchain

Previously, supply chains were moderately easy and simple because commerce was local, but now it's done globally, which makes it incredibly unpredictable. Due to globalization, in between the parties (clients, vendors and the suppliers) may get some more days to be processed, when the review of the contracts are done by the brokers and the lawyers comes with the additional for the delay and the cost. It is considered as unsafe of the goods that are passing through several places geographical locations (international / national) to the destinations that are done with the agreements [20]. It is very hard for tracing where the goods are coming from and where it is as, as those documents about the details may be forged or lost. Now, it is exceptionally for clients/buyers to really gather the information or the products value and the origin of the items, in the supply chain system, there may be lack in the product transparency.

Due to high complexity and lack of transparency in the current supply chain, business people are anxious to explore the possibilities of blockchain [2] technology to transform the supply chain and logistic industry [21]. The records about the digital data [22] or the events are stored in a Distributed Ledger called the Block Chain [2]. It is a database that contains transactions details, information \& records called blocks. These blocks hold incorruptible trust due to its highly secured nature. It offers a compelling solution by combining accessibility with security and privacy. 
In this Globalized world, it is a difficult process to do the supply chain and it likes to be critical compared to others. Today there is a significant amount of trapped value in logistics, mostly stemming from the competitive and fragmented nature of the logistics industry. This frequently makes low transparency, data warehouse, unstandardized processes, and different levels of technology appropriation.

\subsection{Trackability and transparency}

Adopting blockchain [23] in supply chain could support trust, enhanced transparency, and predictability by enabling clients to track where a shipment/ order is at any given time [14].

\subsection{Automation}

Use of smart contracts will enable companies to automate their purchasing process, which leads to cutting costs and saving time. Smart contract will also improve the transaction flow and security in the supply chain.

\subsection{Accessibility}

Ulilizing blockchain [2] dealers can store their product origin, place of storage, authenticity [24], product certificates and record, etc. on a single ledger. All of the important information being in one place will make accessibility data much more easier, which not only create more transparency in the supply chain but also helps in decreasing the amount of frauds and goods robbery that happens.

\subsection{Security}

Since a blockchain [3] is an unchanging distributed ledger, changes in ownership and possession of goods at any point could be entered into the ledger permanently and instantaneously. As the blockchain technology is cryptographically secured and is decentralized, shipping, possession and ownership of data could be better protected from altering or hacks.

\subsection{Quick payments}

Implementing blockchain [2] technology to the payment system could help in reducing grating in commercial financing, accordingly disposing exchaning debate.

\subsection{Saves cost and time}

Transport suppliers will have the capacity to information about availability of storing capacity and routes, which will decrease transport costs and time. Clients can know the origin of the products, manufacturer, date, time, etc,.

\section{SEQUENTIAL DIAGRAM}

Ethereum Blockchain Network [3] is used to verify temperature, tracking shipment data recorded listed in the front-end. Smart contracts written in the contract oriented programming language (solidity), [8] run in a virtual machine, called Ethereum $[11,12,25]$. The Figure 7 show sequential diagram the blockchain.

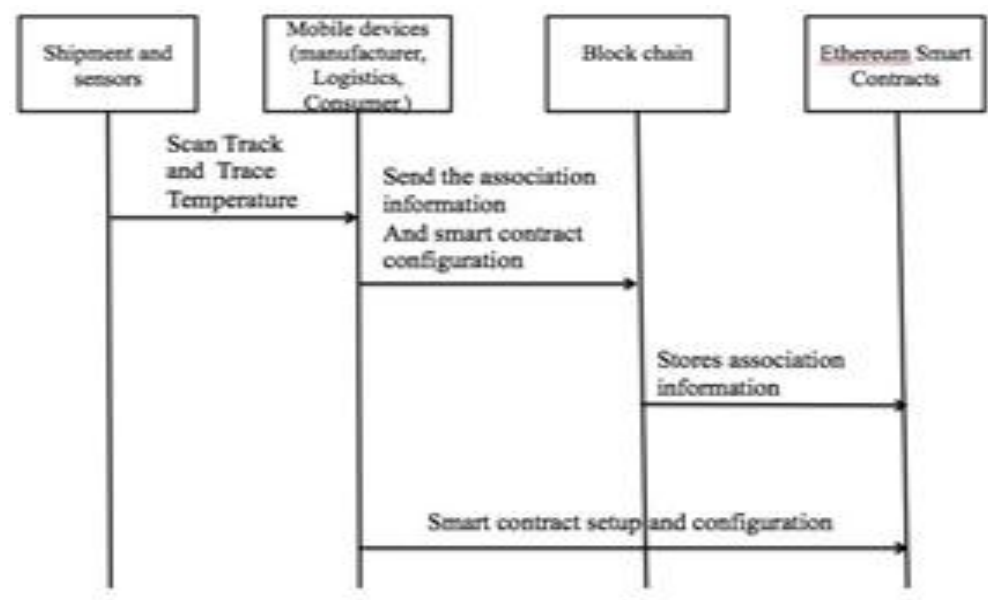

Figure 7. Sequential diagram 
Virtual Machine (EVM) giving power to the verification of data by smart contracts.

a. Smart Contract: is give out for each new shipment, being responsible for making certain the doing as requested of temperature data, tracking shipment data that is connected with the shipment.

b. Mobile Devices: Devices used by the end-users to register new shipments and track/send records of temperature data to the computer application [15].

c. Sensors: sensitive devices able to exist together with Zigbee technology configured to send data in a fixed polling space times between to a Mobile Device.

\section{TECHNICAL DETAILS}

In the back-end, the temperature, tracking shipment doing as requested made certain by smart contracts written with Solidity, a high-level language designed to compile code for EVM. Each and every product with the groups or the newly agreed shipments always has the particular requirements for the temperature. The GDP compliance requirements ensured to do the smart contract for tracking are deployed and configured.

The changes occurred in the smart contracts and the participation of the $[11,12]$ Ethereum networks are done by the Ethereum nodes [26], that initiates the new contracts functions. The communication by the Ethereum nodes by the HTTP (Hypertext Transfered Protocol) over the JSON (JavaScript Object Notation). The ranges of the temperature are verified by the smart contracts and are storing the verified outputs with the hash values in the smart contracts.

The encoding and the decoding for the Android clients for communication with the PC, REST (Representational State Transfer), API (Application Programming Interface)[27] are done by the using the JSON [9]. The users with the mobile phones register for their every new shipment with all their regulatory, in which the contract for every new shipments are created. The recent updates of the temperature records by the Zigbee to the PC or the devices should be allowed by the API. The awareness about the contract results should be known to both sender and the receiver, moreover they should be granted permission to access the measurement of the temperature and the tracability, mainly by using the Graphical Visualization.

The Back-end offered by API can be used in different front-end applications in addition to a smartphone or tablet. For example, one could use a Web application in word used for joining other words, statements with the mobile devices to register doing has requested data of new shipments and verify their separate states on the run. Therefore, one could help from quicker ways to input doing as requested data in contrast with a smartphone or tablet. However, the logistics managing general condition has need of a high mobility of devices reading the sensors, or to register one or many number barcodes at several points of the end-to-end process. Temperature data, tracking location data, preventing damage data is on condition that by IoT sensors by Zigbee device that can be placed in strategical points of the shipment. The sensor has both identification and sensing power which allows to exchange ideas the right in details time to temperature measuring, tracking shipment in specific points.

Temperature looking and tracking shipment location at is started point with the Android client. To start the process, a sensor device needs to be within range. As a first step, a track-and-trace number, which is representatively discovered on the packet, has to be connected with the MAC-address of the sensor device. Since both, track-and- trace number and MAC-address are barcodes, respectively QR-codes, the Android client captures both with its camera. After this process, the Android client starts via Zigbee the temperature measurements and tracking location on the sensor device, and sends the track- and-trace number/MAC-address association to the computer. The sensor also stores the track-and-trace number in case no computer access is provided. Thus, a sending package that has been, always has an association between its MAC-address and the current track-and-trace number. The computer stores the association and makes create existence, broadcasts the smart contract, and stores the smart contract ID on the sensor device. Now the sensor device can be placed inside the products packet. The sensor device is recording every 10 minutes the temperature and stores it in the internal memory on the Zigbee sensor device.Scanning the track and trace number after receiving the packet at the destination. The Android client requests the MAC-Address from the computer to connect to the sensor device [15]. Then the Android client automatically downloads all temperature data, tracking location details and sends it to the smart contract. Once the smart contract checks the temperature, track location anyone interested in that smart contract can verify if the temperature, track was within its specifications directly on the $[11,12]$ Ethereum blockchain $[11,12]$. Thus, the sender will be notified immediately on such result. 


\section{SUMMARY, CONCLUSIONS, AND FUTURE WORK}

Many financial-related start-ups are looking into block chain-based answers in order to get changed to other from the Government controlled organization and amount made less gives idea of price [26]. However, block chains used in other areas as well as IOT and other start-ups working in non-financial areas. Ultimately, the startup rate, the rate of success in the block chain technology, that are both in the Public and the Private applications explain that, the clients technically able to contact and their characteristic, moreover they are having the advantages with the practical exploitation.

\section{REFERENCE}

[1] Amool Sudhan, Manisha J Nene, "Employability of blockchain technology in defence applications,"International Conference on Intelligent Sustainable Systems (ICISS), Conference Paper, Publisher: IEEE, 2017.

[2] Satyabrata Aich, Sabyasachi Chakraborty, Mangal Sain, Hye-in Lee, Hee-Cheol Kim, "A Review on Benefits of IoT Integrated Blockchain based Supply Chain Management Implementations across Different Sectors with Case Study,"21st International Conference on Advanced Communication Technology (ICACT), 2019.DOI: 10.23919/ICACT.2019.8701910.

[3] Si Chen, Rui Shi, Zhuangyu Ren, Jiaqi Yan, Yani Shi, Jinyu Zhang "A Blockchain-Based Supply Chain Quality Management Framework," Published in: 2017 IEEE 14th International Conference on e-Business Engineering (ICEBE), 2017. DOI: 10.1109/ICEBE.2017.34.

[4] Randhir Kumar, Rakesh Tripathi, "Traceability of counterfeit medicine supply chain through Blockchain," Published in: 11th International Conference on Communication Systems \& Networks (COMSNETS), 2019. DOI: 10.1109/COMSNETS.2019.8711418.

[5] Shangping Wang, Dongyi Li, Yaling Zhang, Juanjuan Chen, "Smart Contract-Based Product Traceability System in the Supply Chain Scenario," IEEE Access: Volume: 7, 2019. DOI:10.1109/ACCESS.2019.2935873.

[6] Benhe Gao, Qian Zhou, Shigang Li, Xinglu Liu, "Everledger A Real Time Stare in Market Strategy for Supply Chain Financing Pledge Risk Management,"2018 IEEE International Conference on Industrial Engineeringand Engineering Management (IEEM), Conference Paper, Publisher: IEEE, 2018.

[7] Yonggui Fu, JianmingZhu, "Big Production Enterprise Supply Chain Endogenous Risk Management Based on Blockchain,"IEEEAccess, Volume 7, Journal Article, Publisher: IEEE, 2019.

[8] Michael Mylrea, Sri Nikhil Gupta Gourisetti, "Blockchain for Supply Chain Cybersecurity, Optimization and Compliance,"2018 Resilience Week (RWS), Conference Paper, Publisher: IEEE, 2018.

[9] Mitsuaki Nakasumi, "Information Sharing for Supply Chain Management Based on Block Chain Technology, 2017 IEEE 19th Conference on Business Informatics (CBI), Volume: 01, Conference Paper, Publisher: IEEE, 2017.

[10] Sidra Malik, Salil S. Kanhere, Raja Jurdak"ProductChain: Scalable Blockchain Framework to Support Provenance in Supply Chains,"2018 IEEE 17th International Symposium on Network Computing and Applications (NCA), Conference Paper, Publisher: IEEE, 2018.

[11] Sandi Rahmadika, Bruno Joachim Kweka, Cho Nwe Zin Latt, Kyung-Hyune Rhee, "A Preliminary Approach of Blockchain Technology in Supply Chain System,"2018 IEEE International Conference on Data Mining Workshops (ICDMW), Conference Paper, Publisher: IEEE, 2018.

[12] Miguel Pincheira Caro, Muhammad Salek Ali, Massimo Vecchio, Raffaele Giaffreda, "Blockchain-based traceability in Agri-Food supply chain management: A practical implementation,"2018 IoT Vertical and Topical Summit on Agriculture - Tuscany (IOT Tuscany), Conference Paper, Publisher: IEEE, 2018.

[13] Thomas Bocek, Bruno B. Rodrigues, Tim Strasser, Burkhard Stiller, "Blockchains everywhere - a use-case of blockchains in the pharma supply-chain,"2017 IFIP/IEEE Symposium on Integrated Network and Service Management (IM), Conference Paper, Publisher: IEEE, 2017.

[14] T. A. Alhmiedat and S. H. Yang, "A ZigBee-based Mobile tracking system through wireless sensor networks,"International Journal of Advanced Mechatronic Systems, vol. 1, pp.63-70, 2008.

[15] R. K. Sharma, et al., "Android interfacebased GSM home security system,"Issues and Challenges in Intelligent Computing Techniques (ICICT), 2014International Conference on. IEEE, 2014.

[16] J. Blumenthal, et al., "Weighted centroid localization in ZigBee-based sensor networks," Folien IEEE International Symposium on Intelligent Signal Processing, WISP, Madrid, Spain, 2007.

[17] Kristjan Kuhi, Kati Kaare, Ott Koppel, "Ensuring performance measurement integrity in logistics using blockchain,"2018 IEEE International Conference on Service Operations and Logistics, and Informatics (SOLI), Conference Paper, Publisher: IEEE, 2018.

[18] Jing Hua, Xiujuan Wang, Mengzhen Kang, Haoyu Wang, Fei-Yue Wang, "Blockchain Based Provenance for Agricultural Products: A Distributed Platform with Duplicated and Shared Bookkeeping,"2018 IEEE Intelligent Vehicles Symposium (IV), Conference Paper, Publisher: IEEE, 2018.

[19] Aparna Ramalingaiah, ThaniyaSulthana, "Study of Blockchain with Bitcoin based Fund Raise Use case using Laravel Framework,"2018 3rd International Conference on Computational Systems and Information Technology for Sustainable Solutions (CSITSS), Conference Paper, Publisher: IEEE, 2018.

[20] Adnan Imeri, Djamel Khadraoui, "The Security and Traceability of Shared Information in the Process of Transportation of Dangerous Goods,"2018 9th IFIP International Conference on New Technologies, Mobility and Security (NTMS), Conference Paper, Publisher: IEEE, 2018.

[21] Guido Perboli, Stefano Musso, Mariangela Rosano, "Blockchain in Logistics and Supply Chain: A Lean Approach for Designing Real-World Use Cases,"IEEE Access, Volume: 6, Journal Article, Publisher: IEEE, 2018. 
[22] Weizhi Meng, Elmar Wolfgang Tischhauser, Qingju Wang, Yu Wang, Jinguang Han, "When Intrusion Detection Meets Blockchain Technology: A Review,"IEEE Access, Volume: 6, Journal Article, Publisher: IEEE, 2018.

[23] Shuang Su, Ke Wang, Hyong S. Kim, "Smartsupply: Smart Contract Based Validation for Supply Chain Blockchain,"2018 IEEE International Conference on Internet of Things (iThings) and IEEE Green Computing and Communications (GreenCom) and IEEE Cyber, Physical and Social Computing (CPSCom) and IEEE Smart Data (SmartData), Conference Paper, Publisher: IEEE, 2018.

[24] Mark Kim, Brian Hilton, Zach Burks, Jordan Reyes, "Integrating Blockchain, Smart Contract-Tokens, and IoT to Design a Food Traceability Solution,"2018 IEEE 9th Annual Information Technology, Electronics and Mobile Communication Conference (IEMCON), Conference Paper, Publisher: IEEE, 2018.

[25] Leonor Augusto, Ruben Costa, José Ferreira, Ricardo Jardim-Gonçalves, "An Application of Ethereum smart contracts and IoT to logistics,"2019 International Young Engineers Forum (YEF-ECE), Conference Paper, Publisher: IEEE, 2019.

[26] Raja Jayaraman, Fatima AlHammadi, Mecit Can Emre Simsekler, "Managing Product Recalls in Healthcare Supply Chain," 2018 IEEE International Conference on Industrial Engineering and Engineering Management (IEEM), Conference Paper, Publisher: IEEE, Year: 2018.

[27] Sharma, Rupam Kumar, et al. "Android interfacebased GSM home security system,"Issues and Challenges in Intelligent Computing Techniques (ICICT), International Conference on IEEE, 2014. 